\title{
El papel de la cultura del honor, del sexismo y de los celos en la respuesta a la infidelidad de la pareja
}

\section{The role of culture of honour, sexism and jealousy in response to partner infidelity}

\author{
Jesús María Canto Ortiz, Pilar Moreno Jiménez, Fabiola Perles Novas y Jesús San Martín García
}

Departamento de Psicología Social, Antropología Social, Trabajo Social y Servicios Sociales, Facultad de Psicología, Universidad de Málaga, España

Disponible online 30 de abril de 2012

\begin{abstract}
En esta investigación han participado 170 estudiantes universitarios ( 80 hombres y 90 mujeres). El propósito de este trabajo fue analizar si la cultura del honor, el sexismo y el grado de celos influían en el tipo de infidelidad (sexual o emocional) que más afectaba a los sujetos. Los participantes respondieron a seis dilemas en los que se les planteaban el tipo de infidelidad que más les afectan (sexual o emocional), una escala sobre escala sobre cultura del honor, un inventario sobre sexismo y una escala sobre celos. Los resultados mostraron diferencias entre hombres y mujeres sólo en dos de los seis dilemas. Se analizó la influencia de la cultura del honor, del sexismo hostil, del sexismo benévolo y del grado de celos en la preocupación por la infidelidad sexual y la infidelidad emocional. Se obtuvo que las mujeres que se preocupaban más por la infidelidad sexual puntuaron más alto en cultura del honor, sexismo benévolo y celos.
\end{abstract}

Palabras clave: Celos; Infidelidad, Sexismo Hostil; Sexismo Benévolo; Cultura del Honor.

The aim of this study was to investigate whether a culture of honour, sexism and jealousy influenced the type of infidelity (sexual or emotional) experienced by the participants. A total of 170 university students ( 80 men and 90 women) participated. They responded to six dilemmas indicating which type of infidelity most affected them (sexual or emotional) using a culture of honour scale, a sexism inventory and a jealousy scale. There were differences between men and women only in two dilemmas. The influence of a culture of honour, hostile sexism, benevolent sexism and jealousy on sexual and emotional infidelity was analyzed. Women who scored high on a culture of honour, benevolent sexism and jealousy were those who felt most affected by sexual infidelity.

Keywords: Jealousy; Infidelity; Hostile Sexism; Benevolent Sexism; Culture of Honour.

Correspondencia: Jesús M. Canto Ortiz, Departamento de Psicología Social, Antropología Social, Trabajo Social y Servicios Sociales, Facultad de Psicología, Universidad de Málaga, Campus de Teatinos s/n, 29071, Málaga, Spain. E-mail: jcanto@uma.es. Tlf: 952132465. Fax: 952131100. E-mail otros autores: Pilar Moreno Jiménez: mpilar@uma.es, Fabiola Perles Novas: fanovas@uma.es, Jesús San Martín García. sangar@uma.es 
Los celos son una emoción compleja. Surgen ante la sospecha real o imaginada de una amenaza a una relación que se estima valiosa (Pines, 1992). Al ser muy frecuente la infidelidad en las relaciones de pareja, los celos actuarían como una señal de alarma ante una posible amenaza a la relación; de hecho la infidelidad tiene lugar en todas las culturas y en todas las épocas y suele ser la principal causa de divorcio y maltrato conyugal (Buss y Shackerford, 1997). Buss (2000), desde una perspectiva evolucionista, al investigar las diferencias entre los hombres y las mujeres en su forma de experimentar los celos, sostiene que los hombres expresan mayor estrés que las mujeres ante una infidelidad sexual de su pareja y las mujeres se estresan más que los hombres ante una infidelidad emocional. La asimetría sexual en las respuestas ha sido replicada en distintas investigaciones de diferentes culturas, aunque el tamaño de las diferencias entre ambos sexos varía entre las mismas (v.g.: Buss et al., 1999; Buunk, Angleitner, Oubaid y Buss, 1996; Canto et al., 2011). Estos datos han provocado un fuerte debate teórico, ya que frente a la postura evolucionista hay otras teorías que explican estos resultados a partir de teorías de naturaleza sociocultural (Beall y Sternberg, 1993; Eagly, 1987; Harris, 2003; Hupka, 1991).

Según los psicólogos evolucionistas, si los hombres y las mujeres difieren en el tipo de infidelidad que más les afectan, es consecuencia de los problemas adaptativos tan diferentes que ambos sexos han tenido que afrontar durante el curso de la evolución para garantizar el éxito en la supervivencia y la transmisión de sus propios genes (Daly, Wilson y Weghorst, 1982; Symons, 1979). Basándose en la teoría de la inversión parental (Trivers, 1972), sostienen que existen diferencias importantes entre hombres y mujeres en la cantidad de energía que invierten en la reproducción de los genes y en el grado de seguridad de tal inversión: los hombres realizan una menor inversión de energía que las mujeres para la reproducción con éxito de sus genes y es mayor la seguridad en la inversión parental por parte de las mujeres comparada con la de los hombres. Así, mientras que la infidelidad sexual de la mujer amenazaría la certeza de paternidad del hombre, para la mujer la amenaza principal vendría dada por la posibilidad de que el hombre utilice los recursos que debería prestar en el cuidado de la prole, en el cuidado de otra mujer y sus hijos. Así, con el fin de tener un compañero que les garantice los recursos necesarios para el cuidado de su descendencia, las mujeres requieren que su pareja mantenga una fidelidad emocional que asegure los recursos con los que sacar adelante a los hijos.

Las teorías socioculturales (v.g.: Eagly, 1987), sin embargo, mantienen que las diferencias de comportamiento que existen entre los sexos pueden ser ampliamente explicadas por la socialización diferencial que las sociedades patriarcales proporcionan a los dos sexos. Es decir, las diferencias entre hombres y mujeres se deberían no tanto a influencias de carácter evolutivo, sino a los procesos y estructuras sociales que crean y mantienen estas diferencias. Serían producto de las diferentes expectativas que tanto hombres como mujeres atribuyen al comportamiento social apropiado a ambos sexos. Se puede constatar la existencia de normas duales que históricamente han sido utilizadas para permitir y alentar la actividad sexual masculina, mientras se restringía la actividad sexual femenina (Muehlenhard y McCoy, 1991). Desde este proceso de socialización diferencial, la infidelidad sexual femenina puede ser vista como una violación extrema de las normas sociales y, además, más estresante y más molesta para la pareja de la mujer infiel. La infidelidad sexual de las mujeres en sociedades altamente sexistas (Moya, 2004) puede ser percibida como una conducta antinormativa y extremadamente punible, ya que constituye un ataque al honor del hombre que sufre la ofensa por parte de su pareja que disfruta sexualmente con otro hombre y que, por lo tanto, se aleja de los estereotipos de género tradicionalmente atribuidos a la mujer.

La evidencia sobre las tesis evolucionistas acerca de las diferencias sexuales en las respuestas a la infidelidad ha sido cuestionada. Harris (2003) sostiene que si bien existen estudios que utilizando el dilema de elección forzada diseñado por Buss, Larsen, Westen y Semmelroth (1992) encuentran resultados que podrían avalar la hipótesis evolucionista, también existe un número elevado de investigaciones que no obtienen los mismos resultados. DeSteno y Salovey (1996) objetan al dilema de elección forzada diseñado por Buss et al. (1992) que es difícil imaginar que los dos tipos de infidelidad se den de forma excluyente, ya que la infidelidad suele conllevar aspectos sexuales y emocionales. Siguiendo a Bohner y Wänke (2004), en la investigación que presentamos se han utilizado seis dilemas de elección forzada, donde, además de utilizar los dilemas planteados por Buss et al. (1992), se ha hecho explícita la ocurrencia de ambas infidelidades conjuntamente o se ha especificado que tan sólo era posible un tipo de infidelidad: emocional o sexual.

En esta investigación se analiza si una variable ideológica como la cultura del honor incide en las respuestas de los sujetos a la infidelidad que más les afecta. Por cultura del honor se entiende una predisposición a agredir o a reaccionar emocionalmente de forma violenta como forma de defender algo propio (Puente y Cohen, 2003). La cultura del honor hace referencia a un aspecto cultural con un fuerte componente emocional que tiene una gran influencia en las justificaciones y creencias sobre las reacciones consideradas lícitas ante lo que se considere una ofensa al honor. Se han observado diferencias de sexo en torno a la cultura del honor al puntuar los hombres más alto que las mujeres (Shackelford, 2005). El honor atribuido al sexo femenino se centraría más en la vergüenza sexual (virginidad, modestia y restricciones sexuales) y el honor atribuido al sexo masculino en la virilidad, en la potenciación de la familia y en la reputación como hombre duro (López-Zafra, 2008). Las personas que puntúan alto en cultura del honor tienden a justificar y legitimar la violencia en la pareja provocada por los celos (Puente y Cohen, 2003). 
A pesar de que los psicólogos evolucionistas destacan la asimetría de las respuestas de los hombres y de las mujeres (Buss, 2000), existe una elevada variabilidad de respuestas intra e intersexo en los resultados de las distintas investigaciones llevadas a cabo en distintas culturas. Históricamente los distintos modelos de relaciones de pareja han desarrollado variadas concepciones sobre el amor romántico y han establecido distintos modelos normativos de las relaciones entre hombres y mujeres (Hatfield, Rapson y Martel, 2007). Estas han estado influidas por los estereotipos de género asociados a los distintos sexos y por las actitudes sexistas que conforman tales estereotipos (Rudman y Glick, 2008). Por sexismo hay que entender una ideología de género que implica un conjunto de creencias acerca de los roles considerados apropiados para los hombres y las mujeres (Moya, 2004). Según Glick y Fiske (1996), una de las características de las sociedades occidentales actuales es la coexistencia de una antipatía sexista hacia las mujeres con sentimientos positivos hacia ellas. En este tipo de sexismo, al que denominan sexismo ambivalente, se distinguen dos componentes estrechamente relacionados entre sí: el sexismo hostil (SH) y el sexismo benévolo (SB).

Las personas que puntúan alto en $\mathrm{SH}$ asumen que las mujeres son más débiles que los hombres, sin competencias para gobernar las instituciones sociales y peligrosas y manipuladoras debido al poder sexual que ejercen sobre los mismos. El SB implica que los hombres asumen un rol protector con las mujeres, que éstas tienen características positivas pero complementarias de los hombres y se daría una dependencia diádica de los hombres respecto a ellas. Si el SH se caracteriza por una clara antipatía y rechazo hacia las mujeres, sobre todo hacia las que desafían el poder establecido, el SB asume que las mujeres están mejor en determinados roles (tales como en los roles de madre y esposa) porque son más débiles que los hombres. Los sexistas benévolos tienen una buena imagen de aquellas mujeres que se ajusten a un rol sumiso, pero rechazan, como los sexistas hostiles, a las que desafíen el orden establecido. En este estudio se analizará el papel que desempeña el sexismo en las respuestas que emiten los participantes en el tipo de infidelidad que más les afectan.

En anteriores investigaciones (Canto, García-Leiva, Gómez-Jacinto, 2009; Canto et al., 2011) se obtuvo que las personas más celosas tienden a verse más afectadas por la infidelidad sexual. Lo celoso que sea una persona puede influir en el tipo de infidelidad que más les afecte y en el dolor provocado por las dimensiones sexual y emocional de la infidelidad (Barrón y Martínez-Iñigo, 2001).

En este estudio se quiere analizar las respuestas que emiten los hombres y las mujeres en los seis dilemas del tipo de infidelidad (sexual o emocional) que más les afectan, así como evaluar si el grado de aceptación de actitudes sexistas, de los postulados de la cultura del honor y del grado de celoso que son los participantes influyen en el tipo de infidelidad que más les afectan.
Las hipótesis que se plantean son las siguientes:

Hipótesis 1. Se espera obtener un efecto significativo del sexo en las respuestas de los participantes a los seis dilemas presentados: a los hombres les afectará más la infidelidad sexual mientras que a las mujeres les afectará más la infidelidad emocional (Buss, 2000).

Hipótesis 2. Los sujetos más afectados por la infidelidad sexual puntuarán más alto en cultura del honor, especialmente los hombres más preocupados por la infidelidad sexual.

Hipótesis 3a. Las mujeres que puntúen más alto en SB les afectará más la infidelidad emocional, ya que para ellas es muy importante sentirse protegidas y cuidadas por sus parejas y la infidelidad emocional sería una amenaza mayor que la infidelidad sexual.

Hipótesis 3b. Los hombres que puntúen alto en sexismo, tanto en $\mathrm{SH}$ como en $\mathrm{SB}$, se sentirían peor en mayor proporción ante la infidelidad sexual.

Hipótesis 4. Los participantes más celosos se sentirán más afectados por la infidelidad sexual (Canto et al., 2009; Canto et al., 2011).

\section{Participantes}

\section{Método}

Participaron de forma voluntaria 170 estudiantes universitarios (90 mujeres y 80 hombres) de la Facultad de Filosofía y Letras de la Universidad de Málaga. La edad media de los participantes fue $24.51(D T=6.8$; rango de edad entre 18 y 44 años). Tenían pareja el $87.05 \%(N=148)$ y el $88.23 \%(N=150)$ se consideraban heterosexuales.

\section{Instrumentos}

En primer lugar, se les plantearon seis dilemas de elección forzada (Bohner y Wänke, 2004), adaptada al castellano por uno de los autores, donde los participantes debían señalar si les afectaban más la infidelidad sexual o la infidelidad emocional de su pareja. Los sujetos fueron instruidos a pensar en una relación romántica con compromiso que hubiesen tenido en el pasado, tuvieran actualmente o que les gustaría tener en el futuro. Se les solicitó que imaginasen que su pareja estaba interesada (sexual o emocionalmente) por otra persona y debían señalar el tipo de infidelidad (sexual vs emocional) que les provocase más dolor y malestar. El orden de las alternativas de respuesta (emocional $v s$ sexual) fue balanceado dentro del cuestionario. Los dos primeros dilemas fueron diseñados por Buss et al., (1992). El texto de los dilemas fue el siguiente:

Dilema I: Imagine que descubre que la persona a la que ama está interesada por otra persona. ¿Qué le estresaría o molestaría más?

a) Imaginar que su pareja está disfrutando de un encuentro sexual apasionado con esa persona.

b) Imaginar que su pareja está iniciando una unión afectiva profunda con esa persona.

Dilema II: Imagine que descubre que la persona a la que ama está interesada por otra persona. ¿Qué le angustiaría o molestaría más? a) Imaginar que su pareja se ha enamorado de esa otra persona. 
b) Imaginar a su pareja intentando practicar diferentes posiciones sexuales con esa otra persona.

El dilema número tres especificaba que habían tenido lugar los dos tipos de infidelidad:

Dilema III: Imagine que su pareja ha formado una unión afectiva con otra persona y tiene relaciones sexuales con esa persona. ¿Qué le molestaría más?

a) La unión afectiva de su pareja con otra persona.

b) Las relaciones sexuales de su pareja con otra persona.

Los tres dilemas restantes fueron diseñados para excluir la posibilidad de que un tipo de infidelidad implicara también la otra infidelidad (DeSteno y Salovey, 1996; Gómez-Jacinto, Canto y García-Leiva, 2001; Harris y Christenfeld, 1996). El texto fue el siguiente:

Dilema IV: ¿Qué le molestaría o estresaría más?

a) Imaginar a su pareja iniciar una unión afectiva con esa persona, pero sabiendo con toda seguridad que su pareja no mantiene relaciones sexuales con esa persona.

b) Imaginar que su pareja tiene relaciones sexuales con esa persona, pero sabiendo con toda seguridad que ellos no formarán una unión afectiva profunda.

Dilema V: ¿Qué le molestaría o estresaría más?

a) Imaginar que su pareja está todavía afectivamente implicada con su anterior amante, pero no está interesada sexualmente por ella.

b) Imaginar que su pareja está todavía sexualmente interesada por su anterior amante, pero no está enamorada de ella.

Dilema VI: ¿Qué le molestaría o estresaría más?

a) Imaginar que su pareja está implicada afectivamente con otra persona, pero sin posibilidad de implicación sexual en el futuro.

b) Imaginar que su pareja tiene sólo un encuentro sexual una noche con otra persona, sin posibilidad de implicación afectiva en el futuro.

Se calculó una variable denominada dolor ante la infidelidad (en adelante DI) para medir el dolor que provocaban los dos tipos de infidelidades a partir de los seis dilemas utilizados, de tal forma que cuando los participantes señalaron que la infidelidad sexual les provocaba más dolor puntuaba 1 en cada dilema. Así, la puntuación máxima que se podía obtener en esta variable era 6 (cuando al sujeto le provocaba más dolor la infidelidad sexual en todos los dilemas) y la mínima 0 (cuando el sujeto siempre elegía la infidelidad emocional como la más molesta). Se halló la fiabilidad aplicando el test de Guttman, obteniéndose una puntuación de 0.70 .

En segundo lugar, los participantes respondieron a la escala de la cultura del honor de López-Zafra (2007), en adelante $\mathrm{CH}$. Esta escala consta de 17 ítems, con un formato escala Likert (donde 1 significaba "totalmente en desacuerdo" y 6 "totalmente de acuerdo"). El $\alpha$ de Cronbach obtenido en esta escala fue 0.83 .

En tercer lugar, los sujetos respondieron al Inventario de Sexismo Ambivalente de Glick y Fiske (1996), adaptado al castellano (Expósito, Moya y Glick, 1998). Está compuesto por 22 ítems (11 ítems correspondientes al SH y 11 al SB), con un formato escala Likert ( 0 significa "totalmente en desacuerdo" y 6 "totalmente de acuerdo"). Los $\alpha$ de Cronbach fueron de 0.81 (subescala SH) y 0.83 (subescala SB).

En cuarto lugar, respondieron a la escala de celos románticos (White, 1976), adaptada por Montes-Berges (2008), en adelante GC. Se compone de 6 items que miden la existencia de celos románticos por uno de los miembros de la pareja. Esta escala presenta un formato de respuesta doble: ítems 1, 2, 4 y 6 se contestan de 1 "en absoluto celoso" a 7 "muy celoso" y los ítems 3 y 5 de 1 "nunca" a 7 "a menudo". El $\alpha$ de Cronbach fue 0.87 .

Por último, se recogieron ciertos datos personales de los participantes: sexo, edad, orientación sexual y si tenían pareja.

\section{Procedimiento}

Los cuestionarios fueron aplicados de forma colectiva y anónima en las aulas donde los participantes recibían sus clases universitarias. Recibieron instrucciones por parte de los investigadores de contestar individualmente todos los ítems. Se le informó que sus respuestas eran totalmente anónimas y se les garantizaba la confidencialidad absoluta en el manejo de los datos.

\section{Resultados}

Para analizar las respuestas de los hombres y las mujeres ante el tipo de infidelidad que más les afectó en los seis dilemas se utilizó $\chi^{2}$ (ver Tabla 1). En los dos primeros dilemas (dilemas I y II) se observaron diferencias significativas. Aunque fue mayor la proporción de hombres y de mujeres que se vieron más afectados por la infidelidad emocional, hubo más hombres que mujeres afectados por la infidelidad sexual y más mujeres que hombres por la infidelidad emocional. En los dilemas restantes no se apreciaron diferencias significativas: el patrón de resultados es similar en ambos sexos.

\section{Tabla 1}

Preocupación de hombres y mujeres ante la infidelidad sexual y emocional de sus parejas en los diferentes dilemas planteados (en porcentajes).

\begin{tabular}{|c|c|c|c|c|c|}
\hline \multicolumn{6}{|c|}{ Infidelidad } \\
\hline & & Emocional & Sexual & $\chi^{2}$ & $\mathrm{p}$ \\
\hline \multicolumn{6}{|l|}{ Dilema I } \\
\hline & Hombres & 66.7 & 33.3 & 6.51 & .014 \\
\hline & Mujeres & 85.6 & 14.4 & & \\
\hline \multicolumn{6}{|l|}{ Dilema II } \\
\hline & Hombres & 75.6 & 24.4 & 8.61 & .005 \\
\hline & Mujeres & 93.3 & 6.7 & & \\
\hline \multicolumn{6}{|l|}{ Dilema III } \\
\hline & Hombres & 77.8 & 22.2 & 2.28 & .139 \\
\hline & Mujeres & 87.8 & 12.2 & & \\
\hline \multicolumn{6}{|l|}{ Dilema IV } \\
\hline & Hombres & 60.0 & 40.0 & 2.96 & .065 \\
\hline & Mujeres & 74.4 & 25.6 & & \\
\hline \multicolumn{6}{|l|}{ Dilema V } \\
\hline & Hombres & 57.8 & 42.2 & 2.40 & .88 \\
\hline & Mujeres & 71.1 & 28.9 & & \\
\hline \multicolumn{6}{|l|}{ Dilema VI } \\
\hline & Hombres & 64.4 & 35.6 & 1.83 & .126 \\
\hline & Mujeres & 75.6 & 24.4 & & \\
\hline
\end{tabular}


Se llevó a cabo un análisis de correlación entre las variables DI, CH, SH, SB y GC en los hombres (ver Tabla 2) y en las mujeres (ver Tabla 3). En los hombres, DI correlacionó significativamente con $\mathrm{CH}$ y con $\mathrm{GC}$. $\mathrm{CH}$ correlacionó con $\mathrm{SH}$. Y SH y SB correlacionaron entre sí. El análisis de correlación en las mujeres mostró correlaciones significativas entre todas las variables, a excepción de DI con SH.

Tabla 2

Correlaciones entre las variables en hombres.

\begin{tabular}{lccccc}
\hline & 1 & 2 & 3 & 4 & 5 \\
\hline 1. DI & --- & & & & \\
2. CH & $.40^{* *}$ & --- & & & \\
3. SH & .19 & $.39^{* *}$ & --- & & \\
4. SB & .05 & .12 & $.38^{* *}$ & --- & \\
5. GC & $.35^{*}$ & .20 & .18 & .07 & -- \\
\hline
\end{tabular}

Nota: $D I=$ dolor ante la infidelidad; $C H=$ cultura del honor; $S H=$ sexismo hostil; $S B=$ sexismo benévolo; $C G=$ grado de celos. $* p<.05 ; * * p<.01$

Tabla 3

Correlaciones entre las variables en mujeres.

\begin{tabular}{lccccc}
\hline & 1 & 2 & 3 & 4 & 5 \\
\hline $1 . \mathrm{DI}$ & --- & & & & \\
$2 . \mathrm{CH}$ & $.41^{* *}$ & --- & & & \\
$3 . \mathrm{SH}$ & .14 & $.34^{* *}$ & --- & & \\
$4 . \mathrm{SB}$ & $.36^{* *}$ & $.61^{* *}$ & $.51^{* *}$ & --- & \\
$5 . \mathrm{GC}$ & $.30^{* *}$ & $.51^{* *}$ & $.24^{*}$ & $.56^{* *}$ & -- \\
\hline
\end{tabular}

Nota: $D I=$ dolor ante la infidelidad; $C H=$ cultura del honor; $S H=$ sexismo hostil; $S B=$ sexismo benévolo; $C G=$ grado de celos. $* p<.05 ; * * p<.01$

Se aplicó un ANOVA 2x2 (sexo: hombre vs mujer x dilema 1: emocional vs sexual) en las variables DI, $\mathrm{CH}, \mathrm{SH}, \mathrm{SB}$ y GC (ver Tabla 4). Se utilizó el dilema I porque constituye el dilema estándar que se ha utilizado en la mayoría de las investigaciones en este ámbito (Buss et al., 1992). Concretamente, en el ANOVA llevado a cabo sobre DI, los hombres $(M=2.51 ; D T$ $=1.37)$ puntuaron más alto que las mujeres $(M=1.96 ; D T=$ $1.24 ; F(1,166)=4.55 ; p=.035$ y los participantes que eligieron la infidelidad sexual $(M=3.64 ; D T=1.45)$ puntuaron más alto que los que eligieron la infidelidad emocional $(M=.83 ; D T$ $=1.15 ; F(1,166)=118.3 ; p=.001$. Sin que se obtuvieran dife- rencias significativas en la interacción, fueron los hombres que se sintieron más afectados por la infidelidad sexual $(M=4.13$, $D T=1.45)$ los que puntuaron más alto en DI.

En el ANOVA 2x2 (sexo x dilema $I$ ) aplicado sobre $\mathrm{CH}$, se obtuvo que las mujeres $(M=3.32 ; D T=.88)$ puntuaron más alto que los hombres $(M=2.91 ; D T=.57 ; F(1,166)=6.23 ; p=$ .014. Los participantes más afectados por la infidelidad sexual $(M=3.44 ; D T=.76)$ puntuaron más alto que los que se vieron afectados por la infidelidad emocional $(M=2.80 ; D T=.73 ; F$ $(1,166)=15.19 ; p=.001)$. Aunque no se observaran diferencias significativas en la interacción, fueron las mujeres que eligieron la infidelidad sexual como más estresante las que puntuaron más alto $(M=3.78 ; D T=1.04)$.

Se realizó un ANOVA sobre el SH y otro sobre el SB. En lo que respecta al $\mathrm{SH}$, no se obtuvieron diferencias significativas, ni en función del sexo, del dilema, ni en la interacción. En cuanto al SB, las mujeres $(M=3.82 ; D T=1.39)$ puntuaron más alto que los hombres $(M=3.23, D T=1.10 ; F(1,166)=4.61 ; p$ $=.034)$ y los que eligieron la infidelidad sexual $(M=3.94 ; D T=$ 1.29) más elevado que los que eligieron la infidelidad emocional $(M=3.11 ; D T=1.20 ; F(1,166)=9.18 ; p=.003)$. Se obtuvieron diferencias significativas en la interacción, $F(3,166)=$ $5.99 ; p=.016$. Fueron las mujeres que eligieron la infidelidad sexual como más estresante las que puntuaron más alto en $\mathrm{SB}$ $(M=4.58 ; D T=1.49$; prueba Scheffé, $p=.05)$.

En el ANOVA realizado sobre el GC se obtuvieron diferencias significativas en el sexo y en tipo de dilema. Las mujeres $(M=3.13 ; D T=1.40)$ fueron más celosas que los hombres $(M=$ $2.49 ; D T=1.54 ; F(1,166)=5.15, p=.025)$. Y los que eligieron la infidelidad sexual $(M=3.14, D T=1.60)$ fueron más celosos que los que eligieron la infidelidad emocional $(M=2.48 ; p=$ $.98 ; F(1,166)=5.60 ; p=.02)$. No se obtuvieron diferencias significativas en la interacción, pero los más celosos fueron las mujeres que eligieron la infidelidad sexual $(M=3.28 ; D T=$ 1.66).

Para analizar el efecto de $\mathrm{CH}, \mathrm{SH}, \mathrm{SB}$ y GC sobre DI se llevó a cabo un análisis de regresión múltiple para cada uno de los sexos. En el análisis de regresión en la muestra de hombres se obtuvo que las variables introducidas explicaron un $15.6 \%\left(R^{2} c=.130 ; F(1,170)=15.93 ; p=.001\right)$ del DI. Tan sólo resultó significativa la variable $\mathrm{CH}(\beta=.395 ; t=2.47$;

Tabla 4

Medias y desviaciones típicas en DI, CH, SH, SB y GC de los hombres y de las mujeres en función de si les afectó más la infidelidad emocional o la infidelidad sexual.

\begin{tabular}{|c|c|c|c|c|c|c|c|}
\hline & \multicolumn{2}{|c|}{ Hombre } & \multicolumn{2}{|c|}{ Mujer } & \multicolumn{3}{|c|}{$F$} \\
\hline & Emocional & Sexual & Emocional & Sexual & Sexo & Dilema & Sexo x Dilema \\
\hline & $M(D T)$ & $M(D T)$ & $M(D T)$ & $M(D T)$ & $M(D T)$ & $M(D T)$ & \\
\hline DI & $0.90(1.29)$ & $4.13(1.45$ & $0.77(1.02)$ & $3.15(1.46)$ & $4.55^{*}$ & $118.30^{* *}$ & 2.77 \\
\hline $\mathrm{CH}$ & $2.77(0.75)$ & $3.10(0.49)$ & $2.87(0.72)$ & $3.78(1.04)$ & $6.23 *$ & $15.19 * *$ & 2.65 \\
\hline SH & $3.56(1.03)$ & $3.64(1.12)$ & $3.06(1.37)$ & $3.79(1.72)$ & 0.33 & 1.79 & 1.20 \\
\hline SB & $3.15(1.12)$ & $3.31(1.09)$ & $3.07(1.29)$ & $4.58(1.49)$ & $4.61 *$ & $9.18^{* *}$ & $5.99^{*}$ \\
\hline GC & $1.98(0.83)$ & $3.01(1.54)$ & $2.98(1.14)$ & $3.28(1.66)$ & $5.15^{*}$ & $5.60 *$ & 1.71 \\
\hline
\end{tabular}

Nota: $D I=$ dolor ante la infidelidad; $C H=$ cultura del honor; $S H=$ sexismo hostil; $S B=$ sexismo benévolo $; C G=$ grado de celos. $* p<.05 ; * * p=.001$ 
$p=.019)$. El mismo patrón de resultados se obtuvo con la muestra de mujeres. Las variables introducidas explicaron un $15.7 \%$ $\left(R^{2} c=.155 ; F(1,170)=14.79 ; p=.001\right)$ del DI. Resultó significativa la variable $\mathrm{CH}(\beta=.408 ; t=3.84 ; p=.001)$.

\section{Discusión}

En los seis dilemas planteados, sólo se han encontrado diferencias significativas en las respuestas proporcionadas por los hombres y las mujeres en los dos primeros dilemas (Buss, 2000). Fue mayor el número de hombres y de mujeres más afectados por la infidelidad emocional, pero hubo más hombres que mujeres que se sintieron afectados por la infidelidad sexual y más mujeres que hombres afectadas por la infidelidad emocional. La hipótesis 1 se ha verificado parcialmente. Como señala Harris (2005) para corroborar la hipótesis evolucionista es necesario obtener no sólo diferencias intersexos, sino también diferencias intrasexos; es decir, que a la mayoría de los hombres les afectase más la infidelidad sexual y a la mayoría de las mujeres la infidelidad emocional. Partidarios de la tesis sociocultural como Harris (2003) defienden que si bien la evolución ha perfilado en el ser humano la capacidad de sentir celos, esto no significa que haya dado lugar a mecanismos específicos sexualmente diferenciados que harían que los hombres y las mujeres actuasen de forma diferencial antes los distintos escenarios de infidelidad. Los datos obtenidos, cuando se aplicó el ANOVA (sexo x dilema 1) sobre DI, indicaron que los hombres $(M=2.51 ; D T=1.37)$ manifestaron un mayor dolor ante la infidelidad sexual que las mujeres $(M=1.96, D T=1.24)$, sin embargo en ambos casos la puntuación obtenida por ambos sexos es inferior a la puntuación media de la escala $(M=3)$.

Se obtuvieron diferencias significativas en $\mathrm{CH}$ en función del tipo de infidelidad que más afectaba a los participantes y en función del sexo. Tal como se formuló en la hipótesis 2 , fueron los sujetos más afectados por la infidelidad sexual los que puntaron más alto en $\mathrm{CH}$, en comparación con los que fueron más afectados por la infidelidad emocional. Contrariamente a lo obtenido en otras investigaciones (Shackelford, 2005), las mujeres puntuaron más alto que los hombres en $\mathrm{CH}$. Aunque las diferencias no fueron significativas, no fueron los hombres que eligieron la infidelidad sexual los que puntuaron más alto, sino las mujeres afectadas por ese tipo de infidelidad. Fue la puntuación de estas mujeres las que provocó la mayor puntuación de éstas en $\mathrm{CH}$ comparada con los hombres. Las mujeres que puntúan alto en $\mathrm{CH}$ asumirían que el honor femenino estaría asociado a la aceptación de restricciones sexuales, la valoración de la virginidad y la evitación de conductas promiscuas desde el punto de vista sexual, mientras que el honor masculino se delimitaría por la virilidad masculina (López-Zafra, 2008). Estas mujeres con puntuación alta en $\mathrm{CH}$ coincidirían con los hombres con puntuación alta en $\mathrm{CH}$ en mantener una visión similar de las relaciones de pareja y al considerar la infidelidad sexual como más amenazante para sus relaciones de pareja y para su honor.
Respecto al papel desempeñado por el sexismo en el tipo de infidelidad que más les afectó, no hubo diferencias significativas en $\mathrm{SH}$ en función del sexo, ni en función del tipo de infidelidad, ni en la interacción. Que no hubiera diferencias significativas entre hombres y mujeres no concuerda con las investigaciones en las que se obtiene que los hombres puntúan más alto en SH que las mujeres (Glick y Fiske, 1996). Hay que señalar que, contrariamente a la hipótesis 3a, fueron las mujeres que eligieron la infidelidad sexual como más estresante, las que puntuaron más alto en SB. Como en Canto et al., (2011), los datos de esta investigación no apoyan la hipótesis 3a, que sostenía que las mujeres que puntuasen alto en $\mathrm{SB}$ se verían más afectadas por la infidelidad emocional, ya que fueron las mujeres que eligieron la infidelidad sexual las que puntuaron más alto en SB. Se puede asumir que las mujeres que puntúan alto en SB se sentirían muy molestas por la infidelidad sexual de su pareja ya que, al aceptar para ellas el rol tradicional femenino para obtener los beneficios derivados del SB, la infidelidad sexual de sus parejas han sido con mujeres que han transgredido ese rol tradicional y han admitido mantener una relación sexual quizá de naturaleza esporádica. La hostilidad manifiesta hacia determinados tipos de mujeres que no se ajustan al rol tradicional que el sexismo propone para las mujeres, es mantenida tanto por hombres como por mujeres (Moya, 2004).

Como en investigaciones anteriores (Canto et al., 2009; Canto et al., 2011), también fueron las mujeres más preocupadas por la infidelidad sexual las más celosas, aunque las diferencias no fueron significativas. Si se presta atención al sexo, las mujeres puntuaron más alto en GC que los hombres y los participantes que eligieron la infidelidad sexual fueron más celosos que los que eligieron la infidelidad emocional tal como se formuló en la hipótesis 4. La mayor predisposición a ser celoso hace que la infidelidad sexual sea más molesta, independientemente o no de que hubiera implicación emocional.

Los celos están influidos por múltiples factores, incluidos los factores culturales (Hatfield et al., 2007). En los análisis de regresión llevados a cabo en ambas muestras, la $\mathrm{CH}$ es un predictor del DI. A pesar de las posibles influencias evolutivas en el modo en el que hombres y mujeres experimentan los celos, existe una elevada variabilidad intra e intersexos. En esta investigación se ha observado que si una mujer puntúa alto en $\mathrm{CH}, \mathrm{SB}$ y GC es probable que se sienta más molesta por la infidelidad sexual de su pareja. Esta mujer, al puntuar alto en $\mathrm{CH}$, valorará de forma positiva la fidelidad de las mujeres, la baja promiscuidad sexual de sus parejas y no perdonará fácilmente cualquier tipo de infidelidad (López-Zafra, 2008). La elevada correlación que existe en la muestra de las mujeres entre $\mathrm{CH}$ y SB señalaría la estrecha relación que hay entre una visión tradicional de la mujer y los postulados de la cultura del honor. La correlación entre SB y SH y entre $\mathrm{SH}$ y $\mathrm{CH}$ en las mujeres reflejaría que la hostilidad hacia las mujeres se dirigiría hacia 
aquellas que no se ajustan al rol tradicional de esposa y/o madre y las que su comportamiento podría implicar una grave ofensa al honor de los hombres y un peligro para las mujeres tradicionales.

Los celos son una de las variables que inciden en la violencia en las parejas (Echeburúa y Fernández-Montalvo, 2001). Forman parte, para buena parte de la población, del mito romántico que se los concibe como un signo de amor (Yela, 2000) y son utilizados para justificar la violencia en las relaciones románticas cuando afirma el agresor que actúa por celos (Puente y Cohen, 2003). La respuesta que den los hombres y las mujeres ante la infidelidad de su pareja no sólo estaría condicionada por el grado de celos que sientan, sino por la aceptación de actitudes sexistas y de los postulados de la cultura del honor que justificaría la agresión como una respuesta aceptable ante una ofensa. Que exista la propensión de buena parte de los hombres y de una buena parte de las mujeres a sentirse más afectados por la infidelidad sexual cuando son más sexistas y puntúan alto en la cultura del honor, podría estar condicionado respuestas agresivas en la parejas no sólo cuando ha habido infidelidad, sino también cuando la relación se ha acabado y alguno de los miembros de la pareja se siente celoso cuando el otro miembro inicia una nueva relación romántica con otra persona.

Para profundizar en el impacto de las variables ideológicas en las respuestas que expresen los individuos ante la infidelidad de sus parejas, en futuras investigaciones deberán ampliarse el número de sujetos que participen en la investigación, así como el hecho de su pertenencia a distintas culturas en las que se asuman de forma diferencial los postulados de la cultura del honor y del sexismo. Futuras investigaciones deberán ir más allá de la comparación de muestras de estudiantes universitarios y deberán utilizar otros tipos de metodología en las que se introduzcan situaciones provocadoras de celos (Harris y Darby, 2010).

\section{Referencias}

1. Barrón, A. y Martínez-Iñigo, D. (2001). Los celos: una perspectiva psicológica y social. Málaga: Aljibe.

2. Beall, A. E. y Sternberg, R. J. (1993). The psychology of gender. Nueva York: Guilford.

3. Bohner, G. y Wänke, M. (2004). Psychological gender mediated sex differences in jealousy. Journal of Cultural and Evolutionary Psychology, 2, 213-229. http://dx.doi. org/10.1556/JCEP.2.2004.3-4.3

4. Buss, D. (2000). The dangerous passion: Why jealousy is as necessary as love and sex. Nueva York: Free Press.

5. Buss, D., Larsen, R. J., Westen, D. y Semmelroth, J. (1992). Sex differences in jealousy: Evolution, physiology, and psychology. Psychological Science, 3, 251-255. http:// dx.doi.org/10.1111/j.1467-9280.1992.tb00038.x

6. Buss, D. y Shackelford, T. K. (1997). From vigilance to violence: mate retention tactics in married couples. Journal of Personality and Social Psychology, 72, 346-361. http:// dx.doi.org/10.1037/0022-3514.72.2.346

7. Buss, D., Shackelford, T. K., Kirkpatrick, L. A., Choe, J. C., Lim, H. K., Hasegawa, M., Hasegawa, T. y Bennet, K. (1999). Jealousy and the nature of beliefs about infidelity: Test of competing hypothesis about sex differences in the United States, Korea and Japan. Personal Relationships, 6, 125-150. http://dx.doi.org/10.1111/j.1475-6811.1999. $\underline{\mathrm{tb} 00215 . \mathrm{x}}$

8. Buunk, B. P., Angleiter, A., Oubaid, V. y Buss, D. (1996). Sex differences in jealousy in evolutionary and cultural perspective: Test from Netherlands, Germany, and the United States. Psychological Science, 7, 359-363. http://dx.doi. org/10.1111/j.1467-9280.1996.tb00389.x

9. Canto, J. M., García-Leiva, P. y Gómez-Jacinto, L. (2009). Celos y emociones: Factores de la relación de pareja en la reacción ante la infidelidad. Athenea Digital, 15, 39-55.

10. Canto, J. M., Moreno, P., San Martín, J., Perles, F., Moscato, G. y Reyes, R. M. (2011). Celos y sexismo: un estudio comparativo entre una muestra española y una muestra cubana. Revista de Psicología Social, 26, 33-43. http://dx.doi. org/10.1174/021347411794078480

11. Daly, M., Wilson, M. y Weghorst, S. J. (1982). Male sexual jealousy. Ethology and Sociobiology, 3, 11-27. http:// dx.doi.org/10.1016/0162-3095(82)90027-9

12. DeSteno, D. A. y Salovey, P. (1996). Evolutionary origins of sex differences in jealousy? Questioning the "Fitness" of the Model. Psychological Science, 7, 367-372. http:// dx.doi.org/10.1111/j.1467-9280.1996.tb00391.x

13. Eagly, A. H. (1987). Sex differences in social behavior: A social role interpretation. Hillsdale, $\mathrm{Nj}$ : Erlbaum.

14. Echeburúa, E. y Fernández-Montalvo, J. F. (2001). Celos en la pareja: una emoción destructiva. Barcelona: Ariel.

15. Expósito, F., Moya, M. y Glick, P. (1998). Sexismo ambivalente: medición y correlatos. Revista de Psicología Social, 13, 159-169.

16. Glick, P. y Fiske, S. T. (1996). The Ambivalent Sexism Inventory: Differentiating hostile and benevolent sexism. Journal of Personality and Social Psychology, 70, 491-512. http://dx.doi.org/10.1037/0022-3514.70.3.491

17. Gómez-Jacinto, L., Canto, J. M. y García-Leiva, P. (2001). Variables moduladoras de las diferencias de sexo en los celos. Revista de Psicología Social, 16, 293-313. http:// dx.doi.org/10.1174/021347401317351053

18. Harris, C. R. (2003). A review of sex differences in sexual jealousy, including self-report data, psychophysiological responses, interpersonal violence, and morbid jealousy. Personality and Social Psychology Review, 7, 102-128. http://dx.doi.org/10.1207/S15327957PSPR0702_102-128

19. Harris, C. R. (2005). Male and female jealousy, still more similar than different: Reply to Sagarin (2005). Personality and Social Psychology Review, 9, 76-86. http://dx.doi. org/10.1207/s15327957pspr0901_6 
20. Harris, C. R. y Christenfeld, N. (1996). Gender, jealousy, and reason. Psychological Science, 7, 364-366. http:// dx.doi.org/10.1111/j.1467-9280.1996.tb00390.x

21. Harris, C. R. y Darby, R. S. (2010). Jealousy in adulthood. En S. L. Hart y M. Legerstee (Eds.), Handbook of jealousy (pp. 257-271). Oxford: Wiley-Balckwell.

22. Hatfield, E., Rapson, R. L. y Martel, L. D. (2007). Passionate love and sexual desire. En S. Kitayama y D. Cohen (Eds.), Handbook of Cultural Psychology (pp. 760-779). Nueva York: Guilford Press.

23. Hupka, R. B. (1991). The motive for the arousal of romantic jealousy: Its cultural origin. En P. Salovey (Ed.), The psychology of jealousy and envy (pp. 252-270). Nueva York: Guilford Press.

24. López-Zafra, E. (2007). Elaboración de una escala para medir cultura del honor. Revista de Psicología Social, 22, 31-42. http://dx.doi.org/10.1174/021347407779697520

25. López-Zafra, E. (2008). Relación entre cultura del honor e identidad de género: el papel del sexo, edad y nivel de estudios en la predisposición a la violencia. Revista de Psicología, 29, 209-220.

26. Montes-Berges, B. (2008). Tácticas para la resolución de conflictos y celos románticos en relaciones íntimas: adaptación y análisis de las escalas CTS2 y CR. Estudios de Psicología, 29, 221-134. http://dx.doi. org/10.1174/021093908784485138

27. Moya, M. (2004). Actitudes sexistas y nuevas formas de sexismo. En E. Barberá y I. Martínez (Eds.), Psicología y género (pp. 271-294). Madrid: Prentice-Hall.

28. Muehlenhard, C. L., y McCoy, M. L. (1991). Double standard/double bind: The sexual double standard and women's communication about sex. Psychology of Women Quarterly, 15, 447-462. http://dx.doi.org/10.1111/j.1471-6402.1991. tb00420.x
29. Pines, M. (1992). Romantic jealousy. Nueva York: St Martín's Press.

30. Puente, S. y Cohen, D. (2003). Jealousy and the meaning (or nonmeaning) of violence. Personality and Social Psychology Bulletin, 29, 449-460. http://dx.doi. org/10.1177/0146167202250912

31. Rudman, L. A. y Glick, P. (2008). The social psychology of gender. Nueva York: Guilford Press.

32. Shackelford, T. K. (2005). An evolutionary psychological perspective on cultures of honour. Evolutionary Psychology, 3, 381-391.

33. Symons, D. (1979). The evolution of human sexuality. Nueva York: Oxford University Press.

34. Trivers, R. L. (1972). Parental investment and sexual selection. En B. Campbell (Ed). Sexual selection and the descent of man (pp. 136-179). Chicago: Adline.

35. White, G. L. (1976). The social psychology of romantic jealousy. Unpublished doctoral dissertation. Universidad de California, Los Angeles.

36. Yela, C. (2000). El amor desde la psicología social. Ni tan libres, ni tan racionales. Madrid: Pirámide.

Fecha de recepción: 31 de agosto de 2011

Fecha de recepción de la versión modificada: 29 de septiembre de 2011

Fecha de aceptación: 3 de octubre de 2011 